\title{
EndoFLIP: una nueva tecnología
}

\section{EndoFLIP: a new technology}

\section{Albis Hani, ${ }^{*}$ (1) Andrés Delgado, ${ }^{2}$ (c) Janeth Bejarano, ${ }^{2}$ (1) Diana Meza, ${ }^{2}$ (1) Rosangela Ramírez, ${ }^{2}$ (]) Mariano Pinto, ${ }^{2}$} Jeiler Córdoba, ${ }^{2}$ (1) Andrea Buitrago, ${ }^{2}$ (]) Amaury Amaris, ${ }^{2}$ (1) Carolina Ardila. ${ }^{3}$

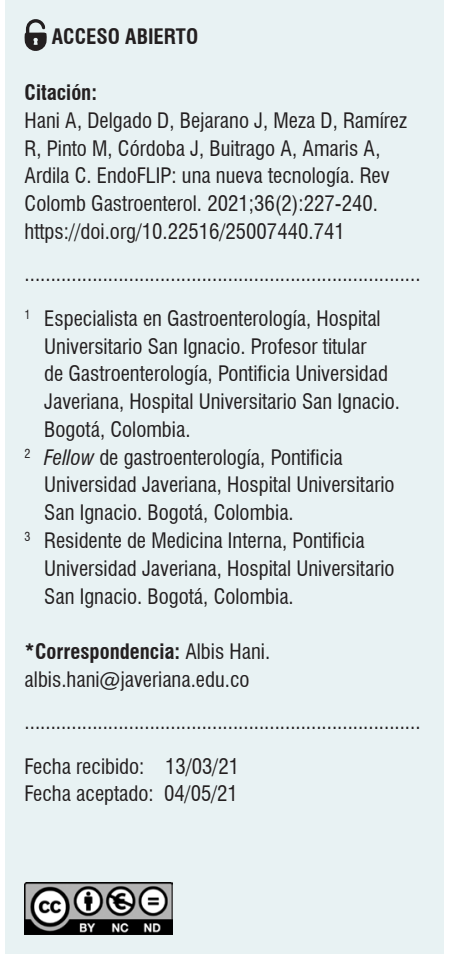

\begin{abstract}
Resumen
Mediante la distensión de un balón con líquido conductor, la sonda de imagen luminal funcional endoluminal (EndoFLIP) evalúa las propiedades biomecánicas como la distensibilidad, volumen, presión e inclusive diámetros de regiones esfinterianas como la unión gastroesofágica, píloro y ano. La mayor evidencia en la utilidad clínica de la EndoFLIP está en los trastornos de motilidad esofágica, principalmente para identificar acalasia cuando la manometría esofágica de alta resolución y otras imágenes no logran diagnosticarla e inclusive, mediante el programa de FLIP 2.0, caracteriza la acalasia en subtipos a partir de patrones de motilidad del esófago distal en respuesta a la distensión. Se ha demostrado recientemente que la EndoFLIP tiene un rol diagnóstico, pronóstico o terapéutico en otras patologías como la esofagitis eosinofilica, reflujo gastroesofágico, gastroparesia, durante la fundoplicatura y dilatación esofágica.
\end{abstract}

\section{Palabras clave}

EndoFLIP, acalasia, impedancia, motilidad, sonda de imagen luminal funcional.

\begin{abstract} troparesis, and during fundoplication and esophageal dilation.

Keywords

EndoFLIP; Achalasia; Impedance; Motility; Functional luminal imaging probe.
\end{abstract}

EndoFLIP evaluates biomechanical properties such as distensibility, volume, pressure, and even diameters of sphincter regions like the gastroesophageal junction, pylorus, and anus, by distending a balloon with a conductive medium. The best evidence of the clinical utility of EndoFLIP is observed in esophageal motility disorders, mainly when identifying achalasia when high-resolution esophageal manometry and other images fail to diagnose it. Even EndoFLIP 2.0 characterizes achalasia into subtypes based on distal esophageal motility patterns in response to distention. Recently, it has been shown that this system has a diagnostic, prognostic and/or therapeutic role in other diseases such as eosinophilic esophagitis, gastroesophageal reflux, and gas-

\section{CONCEPTOS CLAVE}

1. El dispositivo de sonda de imagen luminal funcional endoluminal (EndoFLIP) realiza la medición de presión intrabalón, áreas transversales del esófago y unión gastroesofágica (UGE) por impedancia, e igualmente motilidad por planimetría.
2. Mediante la distensión con balón de la sonda de imagen luminal funcional (FLIP) se produce la apertura de la UGE, que mide su diámetro y distensibilidad; y a su vez hay peristaltismo secundario, que evalúa la motilidad.

3. La técnica FLIP es reconocida en indicaciones como acalasia y esofagitis eosinofílica. Su utilidad es menos 
clara en reflujo gastroesofágico y en la evaluación intraoperatoria de la UGE en fundoplicatura.

4. Mediante la EndoFLIP se puede diagnosticar acalasia y sus subtipos.

5. La EndoFLIP es útil en la evaluación de pacientes con obstrucción al tracto de salida de la UGE, motilidad esofágica inefectiva y contractilidad ausente en quienes clínicamente se sospeche acalasia dentro del espectro de dismotilidad, pero la manometría esofágica de alta resolución (MAR) no es concluyente.

6. Los hallazgos normales en la EndoFLIP pueden ser utilizados para excluir alteraciones motoras esofágicas al tiempo de la endoscopia, lo que podría reducir la necesidad de realizar manometría de alta resolución en la evaluación de algunos pacientes.

7. La respuesta esofágica normal a la distensión en la EndoFLIP durante la endoscopia de vías digestivas altas podría indicar función motora esofágica normal.

8. Dentro de las indicaciones emergentes están su aplicación en gastroparesia y en alteraciones de la función anorrectal.

\section{INTRODUCCIÓN}

La FLIP se basa en el principio de planimetría de impedancia y permite la medición directa de áreas transversales intraluminales (CSA), la evaluación de diámetros intraluminales, perfiles geométricos del lumen y cambios de presión, volumen y distensibilidad; es decir, propiedades biomecánicas de la pared, y brinda una imagen tridimensional de la luz esofágica y de la UGE $(1,2)$. Entonces, la EndoFLIP (sonda de imagen endoluminal funcional) es un sistema que mide en tiempo real el índice de distensibilidad de la unión gastroesofágica (UGE-ID) y el peristaltismo esofágico secundario a través de planimetría de impedancia de alta resolución (3-5).

La evaluación inicial de la planimetría de impedancia como prueba se realizó a finales de la década de 1980 y los primeros estudios se realizaron con los dispositivos en la década de los 1990 (6). A principios de la década de los 2000, varios grupos pioneros liderados por Mittal, Pandolfino y Shaker intentaron abordar varias preguntas sobre el uso de este dispositivo en la evaluación de la distensibilidad y la competencia del esfínter, y sus propiedades biomecánicas. En 2003 se hizo de la planimetría de impedancia algo más práctico, como una opción para evaluar la distensibilidad del esfínter, y condujo a los primeros estudios para la evaluación de la dinámica del esfínter a nivel de la UGE $(7,8)$; también facilitó la medición de la distensibilidad y la detección del punto más estrecho en la UGE.

La Asociación Americana de Gastroenterología (AGA) publicó una revisión de expertos que resume el campo en
2017 (4) y, hasta la fecha, ha habido más de 100 artículos revisados por pares sobre el tema.

\section{DISPOSITIVO Y SISTEMA DE ENDOFLIP}

El dispositivo consta de un catéter de medición, el módulo FLIP 1.0 y el módulo FLIP 2.0 (3) (Figura 1). Existen 2 modelos de catéter FLIP: EF-325, de $8 \mathrm{~cm}$ longitud, con 16 sensores de impedancia planimétrica espaciados cada $5 \mathrm{~mm}$; y EF-322, de $16 \mathrm{~cm}$ con 16 sensores espaciados 1 $\mathrm{cm}$ entre sí. El segmento de medición FLIP se aloja dentro de un balón distensible ubicado en el extremo distal del catéter. En el extremo más distal del balón se ubica un electrodo de estado sólido que mide la presión y en el segmento proximal hay un puerto de infusión a través del cual se perfunde un líquido conductor. El catéter EF-325 proporciona información sobre la distensibilidad de la UGE y CSA, mientras que el catéter EF-322 proporciona patrones de peristaltismo secundario del cuerpo esofágico, además de las métricas de UGE (Tabla 1) $(3,4,9,10)$.

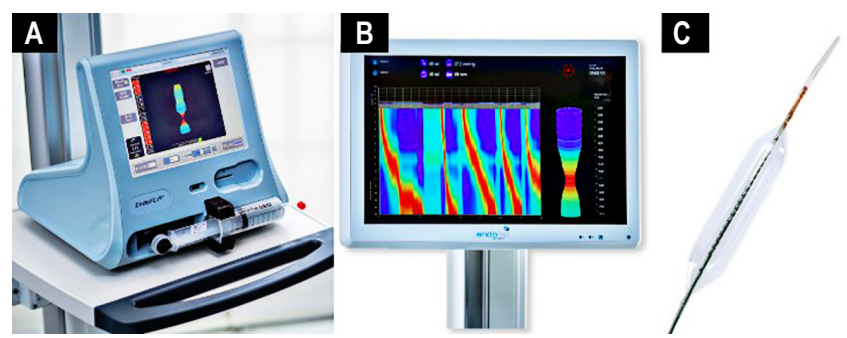

Figura 1. Dispositivo de EndoFLIP. A. Módulo de EndoFLIP con sistema FLIP 1.0. B. Sistema FLIP 2.0. C. Catéter de medición.

\section{INTERPRETACIÓN DE LA ENDOFLIP}

\section{Mediciones y valores normales}

El módulo FLIP 1.0 tiene una jeringa de $80 \mathrm{~mL}$ que funciona mediante una bomba electrohidráulica, que se controla a través de la pantalla táctil de la unidad. La infusión del líquido conductor en el balón permite que los electrodos de excitación, en cada extremo del balón, emitan una corriente eléctrica baja continua y miden el voltaje a través de los sensores de impedancia emparejados (Ley de Ohm: voltaje $=$ corriente $\mathrm{x}$ resistencia). A través del voltaje, el dispositivo FLIP 1.0 calcula las CSA en cada intervalo de pares de sensores, lo que permite estimar el diámetro en 8 o 16 puntos a lo largo del esófago distal. Con el electrodo de presión se mide simultáneamente la presión intrabalón a medida que se realiza la distensión. A partir de la medición simultánea de la presión y las CSA se calcula la distensibilidad. Estos datos se muestran como una representación tridimensional de la luz esofágica (Figura 2) $(3,4,10)$. 
Tabla 1. Tipos de catéter de FLIP, características y protocolos de uso (3)

\begin{tabular}{|lll|}
\hline \multicolumn{1}{|c|}{ Configuración del catéter } & & EF-325 \\
\hline Longitud del balón & $8 \mathrm{~cm}$ & $16 \mathrm{~cm}$ \\
\hline Sensores & 16 separados por $0,5 \mathrm{~cm}$ & 16 separados por $1 \mathrm{~cm}$ \\
\hline Ubicación & $\begin{array}{l}\text { La parte central del catéter se debe ubicar en } \\
\text { la UGE }\end{array}$ & $\begin{array}{l}\text { Se deben ubicar } 2-3 \text { sensores distales por } \\
\text { debajo de la UGE }\end{array}$ \\
\hline Llenado basal & $20 \mathrm{~mL}$ & $30 \mathrm{~mL}$ \\
\hline Tiempo de espera inicial & 15 segundos & 15 segundos \\
\hline Protocolo de llenado & $30 \mathrm{~mL}, 40 \mathrm{~mL}$ y $50 \mathrm{~mL}$ & $40 \mathrm{~mL}, 50 \mathrm{~mL}, 60 \mathrm{~mL}$ y $70 \mathrm{~mL}$ \\
\hline Tiempo de espera por llenado & 30 segundos & 60 segundos \\
\hline Mediciones & UGE-ID y presión intrabalón & $\begin{array}{l}\text { Diámetro UGE-ID, presión intrabalón y patrones } \\
\text { de contractilidad esofágica }\end{array}$
\end{tabular}

Modificado de: Savarino E et al. Am J Gastroenterol. 2020;115(11):1786-1796.

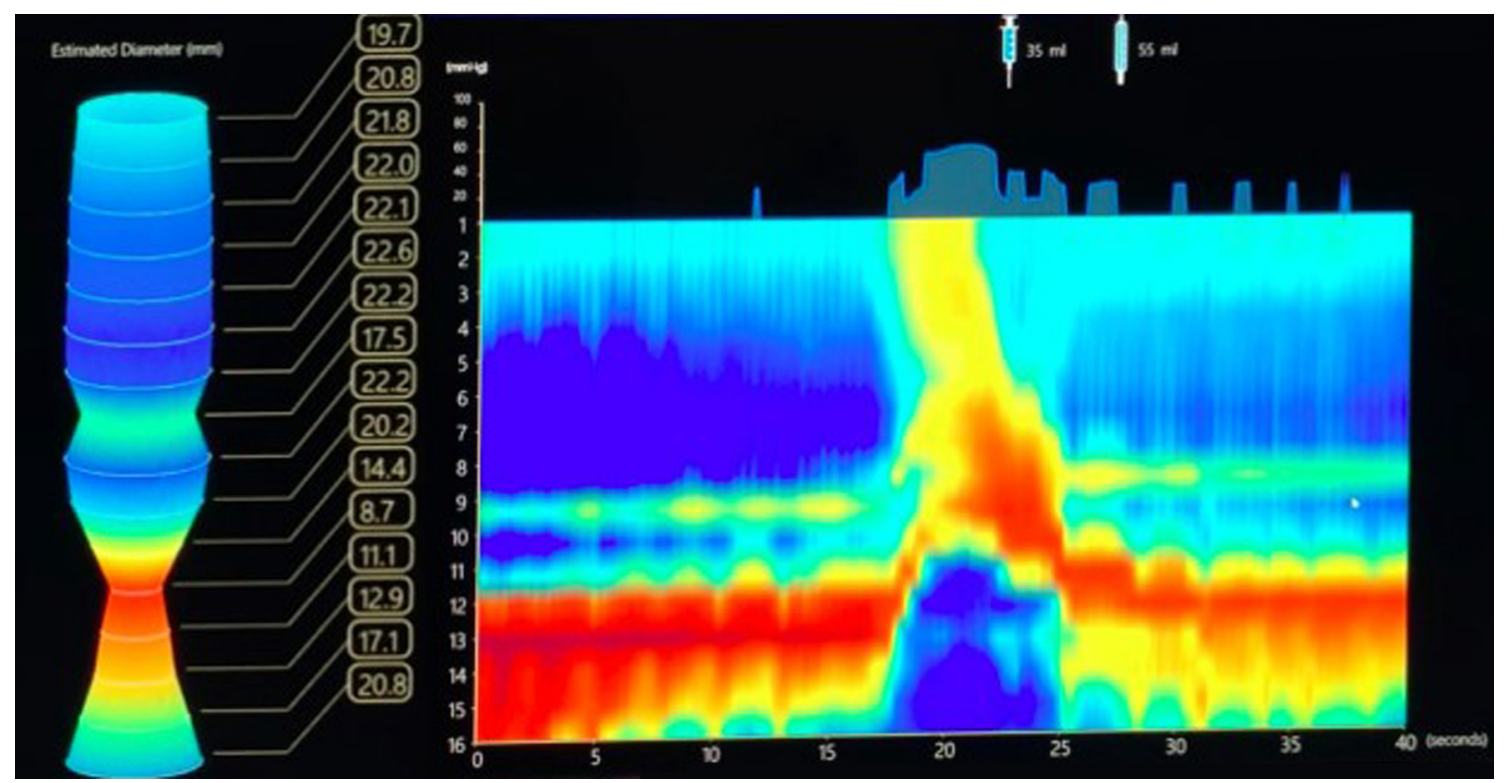

Figura 2. EndoFLIP realizado con catéter EF-322 de $16 \mathrm{~cm}$. A la izquierda, módulo FLIP 1.0 con representación 3D del esófago y sus diámetros. A la derecha, FLIP 2.0 con imagen topográfica del esófago.

En el módulo FLIP 2.0, los datos de los diámetros a lo largo del plano axial del esófago se interpolan y codifican por colores en una escala caliente-fría para generar una topografía FLIP, en la que los diámetros pequeños se representan con los tonos rojos y los diámetros grandes, con tonos azules (Figura 2). Usando el catéter EF-322 en este módulo, se visualizan los cambios de la relación presióndiámetro a lo largo de un continuo espacio-tiempo, útiles para describir patrones de motilidad del esófago distal en respuesta a la distensión $(3,4,11,12)$.

El estudio prospectivo de Carlson y colaboradores (13) evaluó a 20 voluntarios asintomáticos (edad: 23-44; 14 mujeres) con FLIP de $16 \mathrm{~cm}$ colocado a través de la UGE y el esófago distal (y en 8 sujetos también reposicionado en el esófago proximal) durante una endoscopia digestiva alta con sedación (9). Los datos de FLIP se analizaron con 
un programa MATLAB personalizado que generó gráficos de planimetría FLIP y calculó el UGE-ID y las mesetas de distensibilidad del cuerpo esofágico distal y proximal. La contractilidad esofágica inducida por la distensión también fue evaluada. Estos 20 sujetos asintomáticos tenían una UGE-ID $>2,8 \mathrm{~mm}^{2} / \mathrm{mm} \mathrm{Hg}$, un diámetro máximo de UGE $>18 \mathrm{~mm}$ y meseta de distensibilidad en cuerpo esofágico distal $>18 \mathrm{~mm}$, y se evidenció un patrón de contracciones anterógradas repetitivas en respuesta a la distensión.

Entonces, los parámetros normales de la distensibilidad esofágica son UGE-ID $>2,8 \mathrm{~mm}^{2} / \mathrm{mm} \mathrm{Hg}$ y mesetas de distensibilidad $>18 \mathrm{~mm}$. En cuanto a la planimetría, las contracciones anterógradas, que ocurren en un patrón repetitivo con la distensión esofágica con balón, son consideradas fisiológicas (Tabla 2).

\section{Interpretación de valores obtenidos}

Tomando en cuenta los resultados obtenidos luego de la medición, la respuesta contráctil del esófago a la distensión volumétrica se puede formalizar alrededor de 4 categorías distintas (Figura 3):

1. Contracciones anterógradas repetitivas: cuando existen $\geq 3$ contracciones anterógradas consecutivas.

Tabla 2. Interpretación de resultados en EndoFLIP

\begin{tabular}{|c|c|c|c|}
\hline & Normal & Indeterminado & Anormal \\
\hline \multicolumn{4}{|l|}{ Distensibilidad esofágica (FLIP 1.0 y 2.0) } \\
\hline - Índice de distensibilidad de la UGE & $>3 \mathrm{~mm}^{2} / \mathrm{mm} \mathrm{Hg}$ & $2-3 \mathrm{~mm}^{2} / \mathrm{mm} \mathrm{Hg}$ & $>2 \mathrm{~mm}^{2} / \mathrm{mm} \mathrm{Hg}$ \\
\hline - Diámetro de la UGE & $>18 \mathrm{~mm}$ & $13-18 \mathrm{~mm}$ & $<13 \mathrm{~mm}$ \\
\hline \multicolumn{4}{|l|}{ Contractilidad esofágica (FLIP 2.0) } \\
\hline - Contractilidad inducida por la distensión & $\begin{array}{l}\text { Contracciones } \\
\text { anterógradas repetitivas }\end{array}$ & $\begin{array}{l}\text { Patrones contráctiles } \\
\text { indeterminados }\end{array}$ & $\begin{array}{l}\text { Contracciones retrógradas repetitivas } \\
\text { Ausencia de contractilidad }\end{array}$ \\
\hline
\end{tabular}
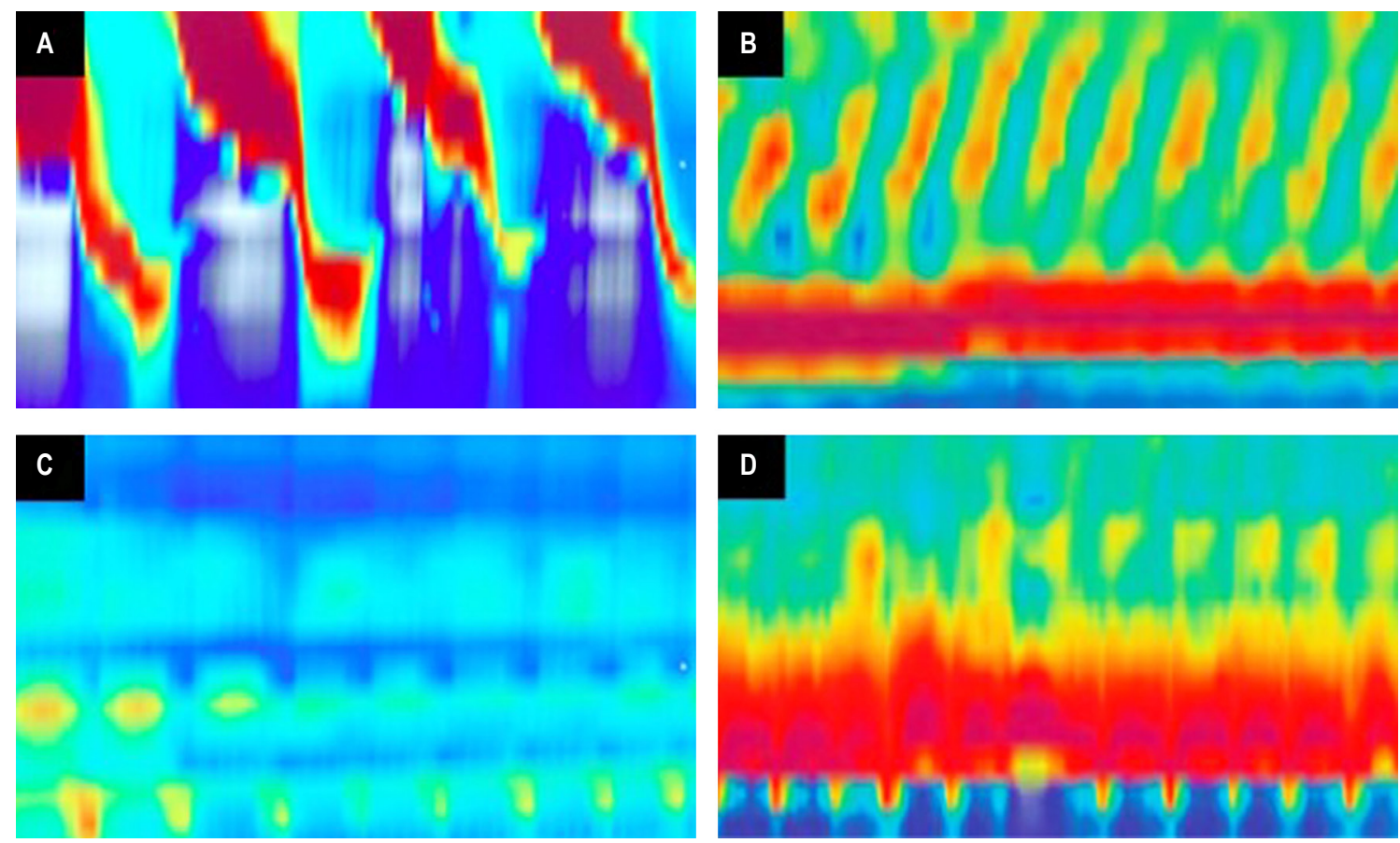

Figura 3. Patrones de contracción esofágica en FLIP 2.0. A. Contracciones anterógradas repetitivas. B. Contracciones retrógradas repetitivas. C. Contractilidad ausente. D. Patrón contráctil indeterminado. 
2. Contracciones retrógradas repetitivas: si hay $\geq 3$ contracciones retrógradas consecutivas, lo cual es una respuesta anormal asociada a menudo con espasmo esofágico, acalasia u obstrucción del flujo de salida de la UGE posquirúrgica.

3. Contractilidad ausente: sugestiva de aperistalsis, acalasia tipo I, esclerodermia y motilidad esofágica inefectiva significativa.

4. Otros patrones contráctiles que no cumplen los criterios para las 3 categorías anteriores, denominados respuesta contráctil disminuida o desordenada, que probablemente representa una forma más leve de disfunción motora.

Estos resultados también pueden utilizarse en estudios comparativos de enfermedades del esófago, como acalasia y esofagitis eosinofílica, lo cual facilitará la aplicación de la planimetría FLIP en la práctica clínica.

La American Gastroenterology Association (AGA) (14) recomienda el uso de la FLIP como complemento de la MAR para el diagnóstico de alteraciones de la motilidad esofágica en pacientes con síntomas esofágicos obstructivos y hallazgos limítrofes en la MAR (recomendación condicional, baja calidad de la evidencia), y en pacientes en quienes el estudio manométrico no pudo ser completado, debido a la falla en la colocación del catéter a pesar de múltiples intentos, por ejemplo.

El estudio de Deprez y colaboradores (15) mostró que la mayoría de los estudios de EndoFLIP identifica a un subgrupo de pacientes con acalasia y es útil en la evaluación intraoperatoria de la eficacia terapéutica en acalasia, pero también consideran el uso de la EndoFLIP en el cuerpo esofágico (planimetría esofágica), al igual que en otras enfermedades esofágicas como la enfermedad por reflujo gastroesofágico (ERGE), esofagitis eosinofílica y en regiones esfinterianas diferentes a la UGE, como el píloro y el canal anal, pero se necesitan estudios confirmatorios.

\section{UTILIDAD CLÍNICA DE LA ENDOFLIP}

\section{Acalasia}

La acalasia es un trastorno primario de la motilidad esofágica que se acompaña de alteración en la relajación del esfínter esofágico inferior (16). El diagnóstico se basa en síntomas como disfagia, regurgitación, pérdida de peso, dolor torácico o pirosis, que deben estar presentes durante al menos 1 año, para no confundirse con pseudoacalasia, como lo indican las guías de Seúl y de la Unión Europea $(17,18)$, más una endoscopia sin obstrucción mecánica del tracto de salida y una MAR compatible con alguno de los subtipos de acalasia definidos en la clasificación de Chicago en su cuarta versión $(16,19)$. Sin embargo, hay pacientes que no toleran la MAR, por lo que la guía del Colegio Americano de Gastroenterología es la primera en plantear la utilidad de la EndoFLIP como herramienta diagnóstica en acalasia (20).

Como lo mencionamos, la EndoFLIP da información importante en el paciente con acalasia, como la distensibilidad del esófago y la UGE, mediante la medición del área de sección transversal de la unión gastroesofágica (CSA-UGE) a través de los sensores de impedancia más la presión $(\mathrm{P})$ dentro del balón, cuyos valores se deben tomar cuando el balón se encuentra distendido con $60 \mathrm{~mL}$ de solución conductora $(2,21)$. La relación CSA-UEG/P es el UGE-ID, cuyo valor normal es de $3,0-9,0 \mathrm{~mm}^{2} / \mathrm{mm} \mathrm{Hg}$. Se considera francamente reducido entre $0-2,0 \mathrm{~mm}^{2} / \mathrm{mm} \mathrm{Hg}$ y en zona gris entre $2,1-3,0 \mathrm{~mm}^{2} / \mathrm{mm} \mathrm{Hg}(21)$. El UGE-ID se reduce en patologías con obstrucción o alteración de la relajación de la UGE, su valor francamente reducido se encuentra en el $91 \%$ de los pacientes con acalasia, pero si se amplía el valor hasta la zona gris mejora su sensibilidad logrando estar presente en el $97 \%$ de los pacientes con acalasia (22). Hay otro parámetro que también tiene relevancia: el diámetro de la UGE, cuyo valor $<16 \mathrm{~mm}$ se observó en el $96 \%$ de los pacientes con acalasia, por lo que tener un UGE-ID $\leq 3,0 \mathrm{~mm}^{2} / \mathrm{mm} \mathrm{Hg}$ más el diámetro de la UGE < 16 confirma por FLIP la presencia de acalasia, ya que no se observan estas características en personas sanas $(9,22)$.

Como se mencionó previamente, la distensión produce contracciones en el cuerpo esofágico, que se miden en la versión FLIP 2.0 (Figura 3) (2,21). Al igual que la MAR, estas contracciones han logrado tipificar la acalasia en 3 tipos, lo cual es bien conocido que tiene valor pronóstico postratamiento $(17,18,20)$. Los patrones de contracciones esofágicas visualizadas por EndoFLIP tienen una sensibilidad/especificidad de $45 \% / 95 \%$ para el diagnóstico de acalasia tipo $1,72 \% / 73 \%$ para el tipo 2 y $93 \% / 82 \%$ para el tipo $3(23,24)$; por lo cual es útil en pacientes cuya MAR no cumple con todos los criterios de Chicago versión 4.0, debido a una peristalsis alterada, pero una presión de relajación integrada (IRP) normal, que con los retos deglutorios y el esofagograma igualmente no logran confirmar la presencia de acalasia (25-27).

Igualmente, la EndoFLIP ha demostrado ser útil en el tratamiento de acalasia, tanto en la fase intraoperatoria como en el seguimiento de los pacientes (28). Durante el procedimiento, ya sea dilatación neumática, miotomía de Heller o miotomía endoscópica por vía oral (POEM), la EndoFLIP es el único método que mide en tiempo real la distensibilidad esofágica, la cual ha demostrado ser útil en predecir una respuesta sintomática definida con Ekcardt $<$ 3 puntos, cuando el valor del UGE-ID es $\geq 3,0 \mathrm{~mm}^{2} / \mathrm{mm}$ $\mathrm{Hg}$, e incluso es mejor que la normalización de la IRP y la 
disminución de la presión del esfínter esofágico inferior medidos en la MAR $(21,28,29)$; además, el trabajo de Campagna y colaboradores demostró que el UGE-ID no se afecta durante la anestesia general (30). De esta forma, la EndoFLIP permite establecer durante el procedimiento, si se requiere, una mayor dilatación o longitud de la miotomía hasta lograr un incremento del UGE-ID; de modo que se logra una mejor respuesta al tratamiento y disminuye el riesgo de reflujo gastroesofágico $(31,32)$.

\section{ERGE}

La función de la barrera antirreflujo con la UGE alterada representa un factor que contribuye en la patogénesis de la ERGE, lo que sugiere la utilidad de la FLIP en la evaluación de la distensibilidad de la UGE para el diagnóstico de la ERGE. Sin embargo, los datos no han demostrado un umbral diagnóstico confiable del UGE-ID en la ERGE.

Como la incompetencia de la UGE puede contribuir con la fisiopatología de la ERGE en algunos pacientes, es razonable sospechar que la FLIP puede ayudar a identificar una mayor distensibilidad de la UGE (es decir, diámetros mayores de la UGE a presiones de distensión más bajas). Sin embargo, los resultados de los estudios han sido inconsistentes. En consecuencia, dado que la EndoFLIP mide la distensibilidad de la UGE, se ha planteado la hipótesis de que este podría mejorar el entendimiento de la fisiopatología de la ERGE y ayudar en el diagnóstico.

Carlson y colaboradores (33) en su estudio consideran que el aumento del UGE-ID contribuye al reflujo. Usando la FLIP, el objetivo fue evaluar la respuesta esofágica a la distensión entre los pacientes sometidos a monitorización del $\mathrm{pH}$ esofágico. La incompetencia de la UGE es un factor importante relacionado con el desarrollo de la ERGE, por lo que la medición de las alteraciones en la distensibilidad de la UGE puede ayudar a caracterizar a los pacientes con ERGE. Este estudio estuvo destinado a evaluar la asociación de la exposición del esófago al ácido con la respuesta esofágica a la distensión entre los pacientes sometidos a evaluación de ERGE con monitorización inalámbrica del $\mathrm{pH}$ esofágico y FLIP, y demostró que, aunque la distensibilidad de la UGE no se asoció consistentemente con la exposición del esófago al ácido, el deterioro en la contractilidad inducida por la distensión se asoció con una mayor exposición del esófago al ácido (34).

Sin embargo, la utilidad de la EndoFLIP en el diagnóstico de la ERGE es controvertida: los estudios realizados para evaluar su utilidad en una población a gran escala han encontrado diferencias en la distensibilidad entre la enfermedad por reflujo erosiva, no erosiva y grupos control. En la ERGE de tipo erosivo, la CSA y el índice de distensibilidad fueron más altos en comparación con el grupo control. Al comparar los resultados de la MAR y la monitorización de $\mathrm{pH}$ de 24 horas, la IRP fue menor en el grupo de reflujo no erosivo que en los otros grupos, y la monitorización del $\mathrm{pH}$ 24 horas mostró una diferencia significativa en todos los grupos; además, debido al alto costo, la EndoFLIP puede no recomendarse a todos los pacientes con ERGE. Puede ser útil en algunos casos, por ejemplo, antes del tratamiento como cirugía antirreflujo, o en ERGE refractaria, ya que se cree que es un método diagnóstico adicional cuando se necesita la información de distensibilidad $(33,34)$.

El UGE-ID en pacientes con ERGE mostró un aumento significativo en comparación con el del grupo de control, independientemente de la presencia de esofagitis erosiva. La medición de la distensibilidad de la UGE utilizando la EndoFLIP es útil en el diagnóstico de la ERGE. Además de las pruebas ambulatorias de $\mathrm{pH}$-impedancia, MAR y endoscopia, la EndoFLIP puede ayudar en el diagnóstico de la ERGE, con base en evidencia objetiva y en la clasificación del subtipo de ERGE.

La FLIP puede también identificar la presencia de hernia hiatal y diferenciar el componente del esfínter esofágico inferior (EEI) del diafragma crural (Figura 4). Se reportó que la distensibilidad del EEI es más alta en presencia de hernia hiatal en comparación con sujetos control asintomáticos (35). Finalmente, usando planimetría FLIP 2.0, Carlson y colaboradores (33) observaron que la exposición esofágica al ácido anormal estuvo asociada con una respuesta contráctil alterada al volumen de distensión del esófago, lo que corrobora el concepto bien conocido de que la exposición del esófago al ácido es dependiente de los mecanismos de depuración de ácido. Sin embargo, los datos sobre la utilidad diagnóstica de la FLIP en la ERGE siguen siendo incompletos, especialmente porque las métricas de la FLIP en la ERGE muestran una superposición considerable con la observada en controles sanos.

En resumen, el papel de FLIP para la evaluación fisiológica y el tratamiento de la ERGE sigue siendo atractivo, pero el nivel de evidencia es bajo y actualmente la FLIP no se recomienda en su enfoque diagnóstico ni el abordaje terapéutico. Se necesitan estudios para corroborar la utilidad de FLIP en la ERGE y desarrollar métricas que ayuden a predecir la gravedad y la respuesta al tratamiento después de los procedimientos antirreflujo.

\section{Disfagia y obstrucción al tracto de salida de la UGE}

La disfagia no obstructiva es uno de los principales síntomas que se estudian para determinar alteraciones en la motilidad esofágica y para lo que se realiza la MAR. Es importante definir cuál es el tipo de trastorno del esfínter esofágico inferior, ya sea acalasia u obstrucción al tracto de salida de la unión esofagogástrica (OTSUEG); se debe 


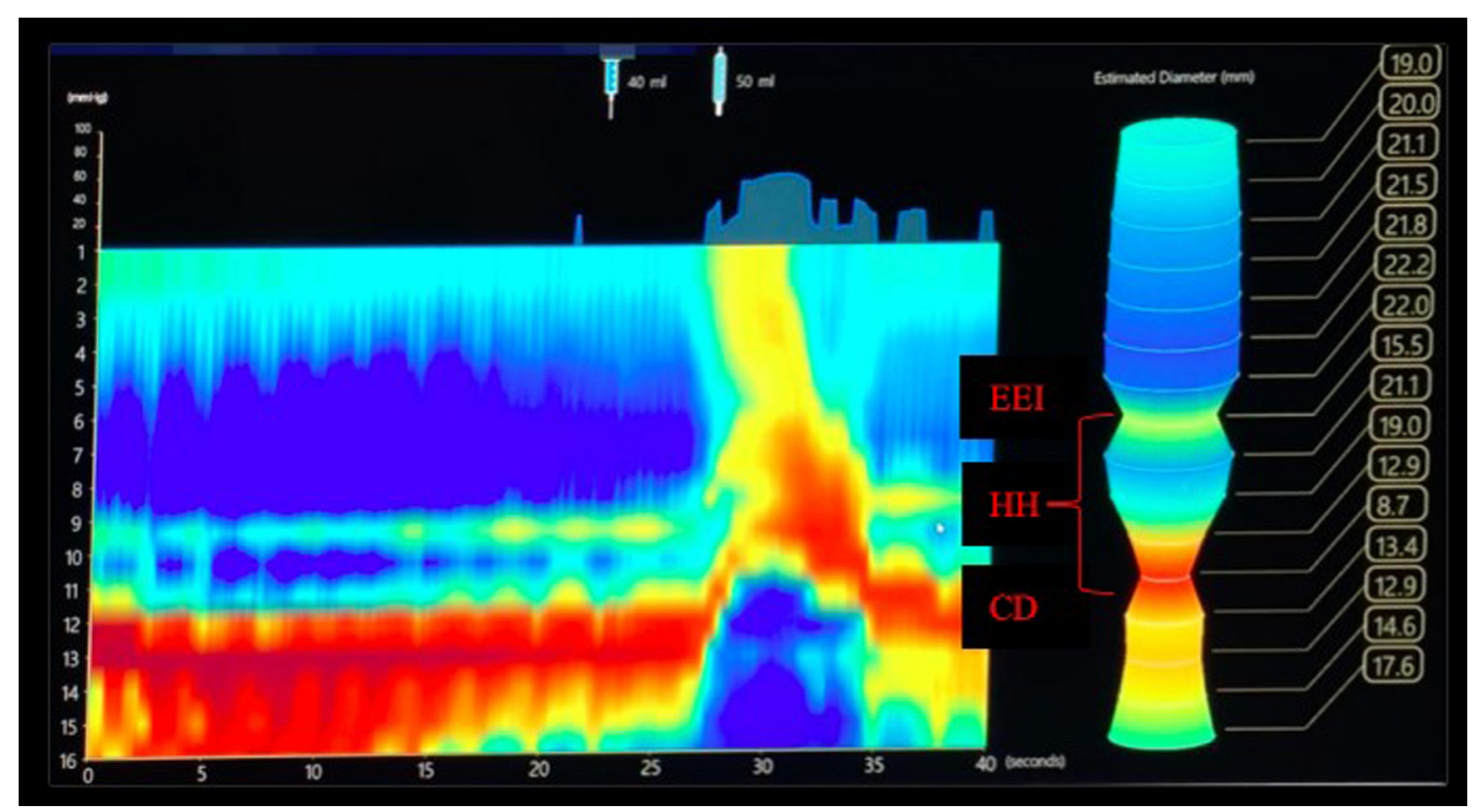

Figura 4. Hernia hiatal en FLIP 2.0. CD: crura diafragmática; HH: hernia hiatal, con diámetro hasta de 21 mm.

tener en cuenta que la MAR tiene sus limitaciones, por lo que la FLIP desempeña un papel importante en la evaluación de la disfagia no obstructiva, puede ser útil en sospecha de acalasia clínica con IRP normal, hallazgos no conclusivos en la MAR y en quienes no toleren la realización de la MAR, entre otros (1).

La OTSUEG es un grupo heterogéneo de trastornos definidos por la clasificación de Chicago 4.0 en los siguientes hallazgos de la MAR: IRP elevada (supina y en vertical), $\geq$ $20 \%$ de degluciones con presión intrabolo en supino elevada y que no cumpla con criterios para acalasia $(19,36)$; estas 2 entidades están dentro de la clasificación de trastornos del esfínter esofágico inferior (37) y se consideran un patrón de motilidad común, pero inespecífico (38); y puede ser causado por una obstrucción funcional, obstrucción mecánica (principalmente en pacientes con antecedente de fundoplicatura, hernia hiatal y estenosis), medicamentos (principalmente opiáceos) o artefactos (36).

El patrón de oro para el diagnóstico de esta entidad es la MAR. Su prevalencia exacta es desconocida, pero se ha reportado que puede variar entre el $3 \%$ y el $21 \%$, con una edad media de presentación entre los 56 y 67 años, en su mayoría mujeres. El síntoma más frecuente es la disfagia, seguido de síntomas típicos y luego atípicos de ERGE; se debe tener en cuenta que el $5 \%$ de los pacientes son asintomáticos, pero se diagnostican por los hallazgos en la MAR. Su significado clínico no es exacto: el impacto en la calidad de vida, la historia natural y el tratamiento óptimo aún no se conocen por completo, pero puede progresar a acalasia, por lo que entonces es una posible representación de una etapa temprana de esta última entidad. Se desconoce qué pacientes pueden progresar a acalasia o cuáles tendrán una resolución temprana (36); por tanto, aún no es claro quiénes se benefician del tratamiento de tipo acalasia (dilatación, miotomía de Heller o POEM) y quiénes se manejarán de forma expectante, con medicamentos o terapia conductual. Debe tenerse en cuenta que hasta el $50 \%$ de los pacientes puede tener una resolución espontánea al tratarse de etiología no mecánica $(37,38)$.

Se cree que la FLIP que utiliza la planimetría de impedancia podría llegar a ser una herramienta prometedora para el diagnóstico y tratamiento de la OTSUEG $(36,37)$, ya que evalúa el diámetro luminal y la correspondiente presión de distensión en el esófago durante la distensión volumétrica (38), que obtiene su valor a través del índice de distensibilidad (37).

Se ha comparado la FLIP con la MAR en pacientes con disfagia y se encontró que la FLIP detecta con mayor precisión trastornos importantes de la motilidad (3); además, analiza la reacción del cuerpo esofágico a la distensión del balón como las contracciones anterógradas repetitivas, contracciones retrógradas repetitivas y contractilidad ausente, que son de utilidad en acalasia como diagnóstico diferencial (36). No obstante, se ha encontrado que la FLIP es útil para identificar pacientes con OTSUEG que tienen más probabilidades de beneficiarse de terapia tipo 
acalasia (aquellos en los que el UGE-ID sea $<2 \mathrm{~mm}^{2} / \mathrm{mm}$ $\mathrm{Hg}$, con una adecuada respuesta posterior al tratamiento) y permite que los pacientes con una distensibilidad $>3 \mathrm{~mm}^{2} /$ $\mathrm{mm} \mathrm{Hg}$ reciban manejo médico con buenos resultados (38); esto sirve como estrategia de seguimiento posterior al tratamiento, en el que se determina que una mejoría en el UGE-ID con un valor $>3 \mathrm{~mm}^{2} / \mathrm{mm} \mathrm{Hg}$ se consideraría normal, lo que muestra una respuesta exitosa al tratamiento y confirma que la eficacia de la modificación del esfínter esofágico inferior está determinada por la mejoría en la distensibilidad del mismo, factor que se evalúa de forma óptima mediante la EndoFLIP (3). Se ha descrito que entre más bajo sea el UGE-ID, será mayor el IRP, lo que puede ser útil para diferenciar los pacientes con obstrucción del flujo de salida más significativa que justifique intervención (37). La capacidad de la EndoFLIP para evaluar tanto la distensibilidad de la UGE como la contractilidad esofágica podría confirmar la relevancia clínica de la IRP anormal aislado en la MAR (3); entonces, esto representa una herramienta de ayuda para mejorar el diagnóstico cuando los hallazgos manométricos son inciertos $y$, adicionalmente, para seleccionar las estrategias de manejo y seguimiento $(3,37,38)$.

\section{EndoFLIP y gastroparesia}

El diagnóstico clínico de gastroparesia puede ser un desafío ya que los síntomas, los patrones y la gravedad están poco correlacionados con el vaciamiento gástrico. Se ha identificado la disfunción pilórica como inusualmente prolongada y las contracciones pilóricas intermitentes denominadas piloroespasmos. Más recientemente, la medición de la distensibilidad pilórica utilizando la EndoFLIP ha sido validada como medición complementaria a la presión del esfínter en gastroparesia. La medición de la distensibilidad pilórica se puede lograr fácilmente colocando una sonda mediante guía radiofluoroscópica a través del endoscopio. Aún no se ha investigado si los anestésicos impactan o no en la distensibilidad pilórica.

En la mayoría de los estudios, la distensibilidad pilórica se ha correlacionado inversamente con el vaciamiento gástrico (15). Además, la distensibilidad pilórica se correlaciona negativamente con los síntomas de gastroparesia, incluidas las náuseas, plenitud gástrica y saciedad temprana. Esto contrasta con la medición del vaciamiento gástrico, que se correlaciona pobremente con los síntomas o la calidad de la vida, lo que sugiere que la distensibilidad pilórica puede ser una herramienta clínicamente relevante en un subconjunto de pacientes con gastroparesia para identificar con mejor precisión uno de los mecanismos subyacentes involucrados en la retención gástrica.

Entre las intervenciones endoscópicas que han demostrado ser efectivas en el tratamiento de la gastroparesia con disfunción pilórica como etiología primaria está la aplicación endoscópica de toxina botulínica, la realización de miotomía peroral endoscópica del píloro (G-POEM) y, en menor medida, la dilatación con balón neumático (39, $40)$. Entre el $30 \%$ y el $50 \%$ de los pacientes con gastroparesia, se ha demostrado una mayor presión y menor elasticidad del esfínter pilórico en comparación con voluntarios sanos; además, las características del píloro observadas con la EndoFLIP aportan información relevante en relación con síntomas, vaciamiento gástrico y predicción de respuesta al tratamiento $(41,42)$.

La primera opción de tratamiento endoscópico en gastroparesia con disfunción pilórica refractaria a los tratamientos farmacológicos y no farmacológicos es la aplicación de toxina botulínica bajo visión directa. Desprez y colaboradores (15) evaluaron la distensibilidad pilórica con EndoFLIP en 35 pacientes con gastroparesia, antes de la aplicación de la toxina botulínica, y encontraron 19 pacientes con distensibilidad pilórica alterada y 16 con distensibilidad pilórica normal; 3 meses después de la aplicación endoscópica de tóxica botulínica, la puntuación sintomática total, calidad de vida, síntomas dispépticos y el vaciamiento gástrico mejoraron en los pacientes con distensibilidad del esfínter pilórico alterada en comparación con aquellos con distensibilidad normal, quienes no tuvieron ninguna mejoría. Lo anterior permite concluir que la evaluación con EndoFLIP predice la respuesta al tratamiento en pacientes con gastroparesia candidatos a la aplicación de toxina botulínica (42).

El G-POEM es una terapia endoscópica reciente de la gastroparesia refractaria. El primer procedimiento se realizó en 2013 y consiste en una disección submucosa del anillo pilórico, la cual ha ganado espacio por su eficacia y seguridad (43). Vosoughi y colaboradores (44) demostraron que la EndoFLIP es una herramienta diagnóstica que permite predecir qué pacientes van a responder a la terapia con G-POEM; al evaluar a 37 pacientes con gastroparesia refractaria de 5 centros, a quienes se les realizó G-POEM y tenían mediciones de EndoFLIP (CSA, presión del balón e índice de distensibilidad), se comparó el éxito clínico al año y el vaciamiento gástrico a los 3 meses, y se encontró que la documentación de la disminución del CSA fue un predictor de éxito posterior a la realización del G-POEM (44).

La dilatación con balón del píloro como tratamiento de los pacientes con gastroparesia refractaria tiene evidencia limitada (40). Sin embargo, en pacientes a quienes se les ha realizado G-POEM o piloroplastia laparoscópica y que persisten sintomáticos, estudios recientes han demostrado un beneficio de la dilatación con balón, dado que la persistencia de los síntomas puede estar relacionada con miotomía incompleta o estenosis por fibrosis. Jehangir y colaboradores (45) incluyeron 13 pacientes con gastroparesia grave refractaria sometidos a pilorotomía y piloroplastia, y 
una posterior dilatación neumática con balón previa valoración de las características pilóricas con EndoFLIP. Se observó que la dilatación mejoró los síntomas en un tercio de los pacientes y se documentó una menor distensibilidad pilórica predilatación con EndoFLIP en los pacientes respondedores (45).

\section{Fundoplicatura y cirugía bariátrica}

Aunque la utilidad diagnóstica de la FLIP en la ERGE puede permanecer controvertida, estudios adicionales han ayudado a evaluar los componentes fisiológicos relacionados con la fisiopatología de la ERGE. Dado que los pacientes con fundoplicatura parecen tener una distensibilidad reducida de la UGE en comparación con los controles, la FLIP puede ser una herramienta útil para ayudar en los procedimientos antirreflujo como la fundoplicatura. Varios estudios demostraron la viabilidad del uso intraoperatorio de FLIP durante la fundoplicatura y una reducción constante de la distensibilidad de la UGE inmediatamente después de la misma. La reducción de la distensibilidad de la UGE después de la fundoplicatura transoral sin incisiónTIF también ha sido reportada.

La EndoFLIP ha demostrado ser un procedimiento complementario útil intraquirúrgico que permite realizar la calibración de la UGE, ya que permite medir en tiempo real el CSA, la disminución de la distensibilidad y el aumento de la presión intraluminal de la UGE como predictor del éxito de la fundoplicatura; además, permite evaluar la longitud de la misma con el objetivo de evitar el exceso de distensibilidad, que es uno de los mecanismos fisiopatológicos de la ERGE, y guiar la modificación intraoperatoria de la técnica en caso de fundoplicatura tipo Nissen muy apretada $(35,46)$, cuantificación que previamente no podía establecerse en la medida objetiva del cierre de la crura; y la realización de la fundoplicatura en la mayoría de los casos se realizaba con una bujía sin una medida más exacta (47). Las mediciones realizadas son la inicial, luego de la intubación, seguida de la movilización o reducción de la hernia, posterior al cierre de la crura y después de la fundoplicatura (48).

Su y colaboradores (49), en su estudio prospectivo que evaluó el uso de EndoFLIP durante el reparo de la hernia hiatal y la fundoplicatura en 175 pacientes con un seguimiento a 24 meses después del tratamiento, encontraron que los pacientes con un UGE-ID final $<2,0 \mathrm{~mm}^{2} / \mathrm{mm}$ Hg presentaron más distensión abdominal y disfagia en comparación con los que tenían un UGE-ID $\geq 2,0 \mathrm{~mm}^{2}$ / $\mathrm{mm} \mathrm{Hg}(p=0,040$ y $p=0,025$, respectivamente), que es más notorio a los 24 meses ( $43 \%$ frente a $12 \%$; $p=0,03$ ). Además, los pacientes con una distensibilidad final entre 2,0 y $3,5 \mathrm{~mm}^{2} / \mathrm{mm} \mathrm{Hg}$ presentaron puntuaciones del índice sintomático menores en comparación con una distensibilidad final $<2,0$ o $>3,5 \mathrm{~mm}^{2} / \mathrm{mm} \mathrm{Hg}(p=0,042)$.

Este dispositivo también ha sido aprobado para guiar la terapia en procedimientos de cirugía bariátrica, lo que permite estandarizar el tamaño y volumen del lumen y, de este modo, mejora los resultados de la técnica en tiempo real (4). Adicionalmente, es de gran importancia en las estenosis después de gastrectomías tipo manga con una incidencia hasta del $4 \%$, que causa síntomas como reflujo, dolor epigástrico, disfagia y regurgitación. La EndoFLIP ha demostrado ser útil para medir la geometría del lumen antes y después de la dilatación, lo que permite establecer una respuesta a la terapia con diámetros posdilatación mayor $(19,9 \pm 2,9 \mathrm{~mm})$ y un UGE-ID de $21,3 \pm 1,0 \mathrm{~mm}^{2}$ / $\mathrm{mm} \mathrm{Hg}(50)$.

\section{Esofagitis eosinofílica}

La esofagitis eosinofílica es una enfermedad crónica del esófago inmunomediada, caracterizada por síntomas esofágicos (predominantemente disfagia e impactación de alimentos) e inflamación eosinofílica (51). Se cree que la inflamación crónica progresa a fibrosis del esófago, que conduce a un estrechamiento luminal y una pérdida de la distensibilidad mural (52). Estas consecuencias de remodelación de la esofagitis eosinofílica son el principal factor determinante de los síntomas y, por lo general, se evalúan mediante endoscopia (53). La prevención y reversión de la remodelación es un objetivo importante del tratamiento (3).

La FLIP se utiliza en esofagitis eosinofílica para evaluar el estrechamiento esofágico y las características mecánicas del cuerpo esofágico, que incluye medir la remodelación esofágica de la fibrosis. La meseta de distensibilidad se identifica con la medición del CSA más estrecho del cuerpo esofágico y la presión intrabalón correspondiente durante la distensión de volumen (2). Los diámetros luminales estrechos se correlacionan con valores de meseta de distensibilidad más bajos (54), que a su vez se correlacionan con la fibrosis de la lámina propia y la aparición de impactación alimentaria. Aunque se puede observar una alteración de la distensibilidad en pacientes con esofagitis eosinofílica de todas las edades, esta disminuye con la edad y la duración de la enfermedad. La distensibilidad deteriorada es parcialmente reversible, especialmente después de un tratamiento exitoso con esteroides o dieta; por el contrario, la distensibilidad no cambia en pacientes con terapia fallida (55).

En el contexto de síntomas persistentes a pesar de la terapia óptima, la evaluación en serie de la distensibilidad y el diámetro luminal utilizando la FLIP podría proporcionar una métrica de resultado objetivo que puede indicar la necesidad de intensificar la terapia. De manera similar, en presencia de remisión histológica con síntomas persisten- 
tes, la FLIP puede revelar otros mecanismos subyacentes a los síntomas clínicos, como una rigidez esofágica aumentada o una distensibilidad reducida debido a la fibrosis esofágica crónica, lo que podría indicar la necesidad de dilatación esofágica. Debido a que esto se puede medir de manera confiable con la FLIP, las mediciones de distensibilidad de la FLIP están ganando importancia como criterios de valoración secundarios en los ensayos de esofagitis eosinofílica y los estudios mecanicistas. Por tanto, las mediciones con EndoFLIP son un complemento fácil para la evaluación del diámetro y la distensibilidad del esófago en la esofagitis eosinofílica (3).

La FLIP puede ser superior a las prácticas actuales de monitorización de la actividad de la enfermedad mediante endoscopia digestiva alta con biopsias porque el muestreo puede ser inconsistente. Se ha encontrado que la fibrosis esofágica medida endoscópicamente es inexacta en comparación con la fluoroscopia (2).

\section{USO TERAPÉUTICO DE LA FLIP}

\section{EsoFLIP}

El EsoFLIP consiste en un balón rígido que realiza la dilatación mediante el llenado del balón controlado por volumen. Los balones de dilatación EsoFLIP están indicados para uso clínico en pacientes adultos para dilatar el EEI en acalasia (dilatación hidráulica) y para dilatar estenosis esofágicas debido a cirugía esofágica, ERGE primaria o radiación.

Aunque el EsoFLIP y la EndoFLIP utilizan la misma tecnología de planimetría de impedancia y tienen varias similitudes, son dispositivos diferentes con aplicaciones clínicas distintas: mientras que el EsoFLIP proporciona dilatación terapéutica, la EndoFLIP es un dispositivo de diagnóstico, como se ha mencionado. Para facilitar la dilatación, el EsoFLIP está disponible en 2 tamaños: a un diámetro de $30 \mathrm{~mm}$ (ES-330) o de $20 \mathrm{~mm}$ (ES-320). Sin embargo, ya que el volumen de llenado del balón es controlado, se puede realizar dilatación a tamaños más pequeños que el tamaño de dilatación máximo (por ejemplo, 16 o 25 $\mathrm{mm}$ ) y es debido a que la visualización en la pantalla en 3D es en tiempo real.

Igualmente, esta tecnología requiere de la visualización endoscópica del sitio que se va a dilatar. Se debe tomar la medida desde las arcadas dentarias, se pasa una guía, se retira el endoscopio y se avanza el catéter de EsoFLIP; si no se utiliza guía, se realiza la medición con el endoscopio desde las arcadas dentarias hasta la zona que se va a dilatar; al retirar el endoscopio se procede a introducir el catéter de EsoFLIP y se confirma su ubicación nuevamente con visualización endoscópica; ambas técnicas se pueden confirmar en la pantalla de la $\operatorname{FLIP}(56,57)$.

La evaluación en tiempo real de la dilatación proporcionada por la planimetría de impedancia con el EsoFLIP, así como el volumen de llenado del balón y dilatador controlado, ofrece varias ventajas potenciales sobre otros dilatadores de balón. Aunque los datos clínicos asociados con este nuevo dispositivo siguen siendo limitados, la seguridad y la viabilidad de la dilatación con balón EsoFLIP se han reportado $(56,57)$.

\section{Ventajas de la dilatación con EsoFLIP}

\section{Dilatación sin fluoroscopia}

La visualización de la cintura en EsoFLIP a través de planimetría de impedancia en tiempo real puede facilitar la realización de la dilatación, en particular para dilataciones de $30 \mathrm{~mm}$, sin necesidad de fluoroscopia. Además, si se desplaza el balón, se puede corregir su posición en tiempo real visualizado en la pantalla.

\section{Control del tamaño (diámetro) de la dilatación}

Se define el tamaño de la dilatación mediante el control del volumen de llenado del balón. La medición en tiempo real en la cintura del balón permite un diámetro de dilatación controlado para generar el efecto deseado. Esto contrasta con el uso de un balón dilatador de un solo tamaño o incluso de varios tamaños que puede requerir el uso de varios balones si no se logra el efecto deseado.

\section{Medición del tamaño de la estenosis y evaluación del efecto de dilatación}

La dilatación con EsoFLIP ofrece la posibilidad de medir el tamaño de la estenosis y de evaluar la respuesta a la dilatación inmediatamente después del procedimiento. Los estudios que utilizan EndoFLIP han demostrado su utilidad para medir el tamaño de la estenosis, particularmente en la esofagitis eosinofílica (55, 57-59). Además, la medición del UGE-ID con la EndoFLIP en la acalasia facilita la evaluación de su respuesta al tratamiento (miotomía o dilatación neumática) con los resultados clínicos en el momento del tratamiento (57, 60, 61). El EsoFLIP 330 también sirve para realizar dilatación de la UGE en pacientes con acalasia y el éxito, que se define como un Eckardt < 3 , se obtuvo en el $63 \%$ de los pacientes, sin mayores eventos adversos $(62,63)$.

\section{Limitaciones de la dilatación con EsoFLIP}

Algunos aspectos del dispositivo pueden limitar la facilidad del procedimiento, como la capacidad para pasar la 
guía a través del endoscopio y que debe retirarse antes de la dilatación, la necesidad de un monitor y el tiempo necesario para llenar y vaciar el balón dilatador. Además, y como era de esperarse debido a la novedad del dispositivo, existen pocos estudios que informen sobre la utlización o eficacia del EsoFLIP (57).

El estudio de Schnurre y colaboradores (63) encontró una buena eficacia tanto en el resultado subjetivo como objetivo del tratamiento a corto plazo después de la dilatación con EsoFLIP en pacientes con acalasia sin tratamiento previo. Esos hallazgos sugieren que el EsoFLIP es una tecnología de dilatación prometedora que debería estudiarse más a fondo en un entorno controlado más grande y con un seguimiento más prolongado.

\section{CONCLUSIÓN}

La EndoFLIP es una nueva tecnología disponible en nuestro país con una amplia utilidad clínica, principalmente como herramienta diagnóstica complementaria, cuando la manometría esofágica con retos deglutorios e imágenes complementarias (esofagograma o tableta de bario) no son suficientes para confirmar trastornos funcionales de obstrucción al tracto de salida de la UGE. Adicionalmente, tiene un rol terapéutico en la dilatación de la acalasia, estenosis esofágica y durante la cirugía de fundoplicatura. Está por determinarse la utilidad clínica en otras patologías como la esofagitis eosinofílica, reflujo gastroesofágico, gastroparesia, entre otras.

\section{REFERENCIAS}

1. Dorsey YC, Posner S, Patel A. Esophageal Functional Lumen Imaging Probe (FLIP): How Can FLIP Enhance Your Clinical Practice? Dig Dis Sci. 2020;65(9):2473-2482. https://doi.org/10.1007/s10620-020-06443-8

2. Donnan EN, Pandolfino JE. EndoFLIP in the Esophagus: Assessing Sphincter Function, Wall Stiffness, and Motility to Guide Treatment. Gastroenterol Clin North Am. 2020;49(3):427-435. https://doi.org/10.1016/j.gtc.2020.04.002

3. Savarino E, di Pietro M, Bredenoord AJ, Carlson DA, Clarke JO, Khan A, et al. Use of the Functional Lumen Imaging Probe in Clinical Esophagology. Am J Gastroenterol. 2020;115(11):1786-1796. https://doi.org/10.14309/ajg.0000000000000773

4. Hirano I, Pandolfino JE, Boeckxstaens GE. Functional Lumen Imaging Probe for the Management of Esophageal Disorders: Expert Review From the Clinical Practice Updates Committee of the AGA Institute. Clin Gastroenterol Hepatol. 2017;15(3):325-334. https://doi.org/10.1016/j.cgh.2016.10.022

5. Donnan EN, Pandolfino JE. Applying the Functional Luminal Imaging Probe to Esophageal Disorders. Curr Gastroenterol Rep. 2020;22(3):10. https://doi.org/10.1007/s11894-020-0749-7

6. Clarke JO, Ahuja NK, Fernandez-Becker NQ, Gregersen $\mathrm{H}$, Kamal AN, Khan A, et al. The functional lumen imaging probe in gastrointestinal disorders: the past, present, and future. Ann N Y Acad Sci. 2020;1482(1):16-25. https://doi.org/10.1111/nyas.14463

7. McMahon BP, Frøkjaer JB, Liao D, Kunwald P, Drewes AM, Gregersen H. A new technique for evaluating sphincter function in visceral organs: application of the functional lumen imaging probe (FLIP) for the evaluation of the oesophago-gastric junction. Physiol Meas.
2005;26(5):823-36.

https://doi.org/10.1088/0967-3334/26/5/019

8. McMahon BP, Frøkjaer JB, Drewes AM, Gregersen H. A new measurement of oesophago-gastric junction competence. Neurogastroenterol Motil. 2004;16(5):543-6. https://doi.org/10.1111/j.1365-2982.2004.00540.x

9. Bianca A, Schindler V, Schnurre L, Murray F, Runggaldier D, Gyawali CP, et al. Endoscope presence during endoluminal functional lumen imaging probe (FLIP) influences FLIP metrics in the evaluation of esophageal dysmotility. Neurogastroenterol Motil. 2020;32(6):e13823. https://doi.org/10.1111/nmo.13823

10. Valdovinos LR, Vela MI. Utilidad de la sonda de imagen luminal funcional (Endoflip) en las enfermedades esofágicas. Acta Gastroenterol Latinoam 2020,50:79-87

11. Carlson DA, Kahrilas PJ, Lin Z, Hirano I, Gonsalves N, Listernick Z, et al. Evaluation of Esophageal Motility Utilizing the Functional Lumen Imaging Probe. Am J Gastroenterol. 2016;111(12):1726-1735. https://doi.org/10.1038/ajg.2016.454

12. Carlson DA, Lin Z, Rogers MC, Lin CY, Kahrilas PJ, Pandolfino JE. Utilizing functional lumen imaging probe topography to evaluate esophageal contractility during volumetric distention: a pilot study. Neurogastroenterol Motil. 2015;27(7):981-9. https://doi.org/10.1111/nmo.12572

13. Carlson DA, Kou W, Lin Z, Hinchcliff M, Thakrar A, Falmagne $S$, et al. Normal Values of Esophageal Distensibility and Distension-Induced Contractility Measured by Functional Luminal Imaging Probe Panometry. Clin Gastroenterol Hepatol. 2019;17(4):674-681.e1. https://doi.org/10.1016/j.cgh.2018.07.042

14. Gyawali CP, Carlson DA, Chen JW, Patel A, Wong RJ, Yadlapati RH. ACG Clinical Guidelines: Clinical Use 
of Esophageal Physiologic Testing. Am J Gastroenterol. 2020;115(9):1412-1428.

https://doi.org/10.14309/ajg.0000000000000734

15. Desprez C, Roman S, Leroi AM, Gourcerol G. The use of impedance planimetry (Endoscopic Functional Lumen Imaging Probe, EndoFLIP ${ }^{*}$ ) in the gastrointestinal tract: A systematic review. Neurogastroenterol Motil. 2020;32(9):e13980. https://doi.org/10.1111/nmo.13980

16. Patel DA, Lappas BM, Vaezi MF. An Overview of Achalasia and Its Subtypes. Gastroenterol Hepatol (N Y). 2017;13(7):411-421.

17. Jung HK, Hong SJ, Lee OY, Pandolfino J, Park H, Miwa H, et al. 2019 Seoul Consensus on Esophageal Achalasia Guidelines. J Neurogastroenterol Motil. 2020;26(2):180-203. https://doi.org/10.5056/jnm20014

18. Oude Nijhuis RAB, Zaninotto G, Roman S, Boeckxstaens GE, Fockens P, Langendam MW, et al. European guidelines on achalasia: United European Gastroenterology and European Society of Neurogastroenterology and Motility recommendations. United European Gastroenterol J. 2020;8(1):13-33. https://doi.org/10.1177/2050640620903213

19. Yadlapati R, Kahrilas PJ, Fox MR, Bredenoord AJ, Prakash Gyawali C, Roman S, et al. Esophageal motility disorders on high-resolution manometry: Chicago classification version $4.0^{\circ}$. Neurogastroenterol Motil. 2021;33(1):e14058. https://doi.org/10.1111/nmo.14058

20. Vaezi MF, Pandolfino JE, Yadlapati RH, Greer KB, Kavitt RT. ACG Clinical Guidelines: Diagnosis and Management of Achalasia. Am J Gastroenterol. 2020;115(9):1393-1411. https://doi.org/10.14309/ajg.0000000000000731

21. Massey BT. Clinical Functional Lumen Imaging Probe Testing in Esophageal Disorders: A Need for Better Quality Evidence. Am J Gastroenterol. 2020;115(11):1799-1801. https://doi.org/10.14309/ajg.0000000000000974

22. Rooney KP, Baumann AJ, Donnan E, Kou W, Triggs JR, Prescott J, et al. Esophagogastric Junction Opening Parameters Are Consistently Abnormal in Untreated Achalasia. Clin Gastroenterol Hepatol. 2021;19(5):10581060.e1. https://doi.org/10.1016/j.cgh.2020.03.069

23. Carlson DA, Lin Z, Kahrilas PJ, Sternbach J, Donnan EN, Friesen L, et al. The Functional Lumen Imaging Probe Detects Esophageal Contractility Not Observed With Manometry in Patients With Achalasia. Gastroenterology. 2015;149(7):1742-51. https://doi.org/10.1053/j.gastro.2015.08.005

24. Carlson DA, Kou W, Rooney KP, Baumann AJ, Donnan E, Triggs JR, et al. Achalasia subtypes can be identified with functional luminal imaging probe (FLIP) panometry using a supervised machine learning process. Neurogastroenterol Motil. 2021;33(3):e13932.

https://doi.org/10.1111/nmo.13932

25. Sanagapalli S, Roman S, Hastier A, Leong RW, Patel K, Raeburn A, et al. Achalasia diagnosed despite normal inte- grated relaxation pressure responds favorably to therapy. Neurogastroenterol Motil. 2019;31(6):e13586. https://doi.org/10.1111/nmo.13586

26. Ang D, Hollenstein M, Misselwitz B, Knowles K, Wright J, Tucker E, et al. Rapid Drink Challenge in high-resolution manometry: an adjunctive test for detection of esophageal motility disorders. Neurogastroenterol Motil. 2017;29(1). https://doi.org/10.1111/nmo.12902

27. Horton A, Jawitz N, Patel A. The Clinical Utility of Provocative Maneuvers at Esophageal High-resolution Manometry (HRM). J Clin Gastroenterol. 2021;55(2):95-102. https://doi.org/10.1097/MCG.0000000000001466

28. Khashab MA, Vela MF, Thosani N, Agrawal D, Buxbaum JL, Abbas Fehmi SM, et al. ASGE guideline on the management of achalasia. Gastrointest Endosc. 2020;91(2):213227.e6. https://doi.org/10.1016/j.gie.2019.04.231

29. Carlson DA, Hirano I. Application of the Functional Lumen Imaging Probe to Esophageal Disorders. Curr Treat Options Gastroenterol. 2017;15(1):10-25. https://doi.org/10.1007/s11938-017-0116-4

30. Campagna RAJ, Carlson DA, Hungness ES, Holmstrom AL, Pandolfino JE, Soper NJ, et al. Intraoperative assessment of esophageal motility using FLIP during myotomy for achalasia. Surg Endosc. 2020;34(6):2593-2600. https://doi.org/10.1007/s00464-019-07028-x

31. Attaar M, Su B, Wong HJ, Kuchta K, Denham W, Haggerty SP, et al. Intraoperative impedance planimetry (EndoFLIP ${ }^{\mathrm{rm}}$ ) results and development of esophagitis in patients undergoing peroral endoscopic myotomy (POEM). Surg Endosc. 2020. https://doi.org/10.1007/s00464-020-07876-y

32. Su B, Dunst C, Gould J, Jobe B, Severson P, Newhams K, et al. Experience-based expert consensus on the intra-operative usage of the Endoflip impedance planimetry system. Surg Endosc. 2021;35(6):2731-2742. https://doi.org/10.1007/s00464-020-07704-3

33. Carlson DA, Kathpalia P, Craft J, Tye M, Lin Z, Kahrilas PJ, et al. The relationship between esophageal acid exposure and the esophageal response to volumetric distention. Neurogastroenterol Motil. 2018;30(3):10.1111/ nmo.13240. https://doi.org/10.1111/nmo.13240

34. Lee JM, Yoo IK, Kim E, Hong SP, Cho JY. The Usefulness of the Measurement of Esophagogastric Junction Distensibility by EndoFLIP in the Diagnosis of Gastroesophageal Reflux Disease. Gut Liver. 2020. https://doi.org/10.5009/gnl20117

35. Lottrup C, McMahon BP, Ejstrud P, Ostapiuk MA, FunchJensen P, Drewes AM. Esophagogastric junction distensibility in hiatus hernia. Dis Esophagus. 2016;29(5):463-71. https://doi.org/10.1111/dote.12344

36. Zikos TA, Triadafilopoulos G, Clarke JO. Esophagogastric Junction Outflow Obstruction: Current Approach to Diagnosis and Management. Curr Gastroenterol Rep. 
2020;22(2):9.

https://doi.org/10.1007/s1 1894-020-0743-0

37. Reddy AT, Shimpi RA, Parish A, Niedzwiecki D, Leiman

DA. Predictors of Abnormal Functional Luminal Impedance

Planimetry Findings in Non-mechanical Esophagogastric

Junction Outflow Obstruction. Dig Dis Sci. 2020.

https://doi.org/10.1007/s10620-020-06726-0

38. Triggs JR, Carlson DA, Beveridge C, Kou W, Kahrilas PJ, Pandolfino JE. Functional Luminal Imaging Probe Panometry Identifies Achalasia-Type Esophagogastric Junction Outflow Obstruction. Clin Gastroenterol Hepatol. 2020;18(10):2209-2217. https://doi.org/10.1016/j.cgh.2019.11.037

39. Sullivan A, Temperley L, Ruban A. Pathophysiology, Aetiology and Treatment of Gastroparesis. Dig Dis Sci. 2020;65(6):1615-1631. https://doi.org/10.1007/s10620-020-06287-2

40. Usai-Satta P, Bellini M, Morelli O, Geri F, Lai M, Bassotti G. Gastroparesis: New insights into an old disease. World J Gastroenterol. 2020;26(19):2333-2348. https://doi.org/10.3748/wjg.v26.i19.2333

41. Saadi M, Yu D, Malik Z, Parkman HP, Schey R. Pyloric sphincter characteristics using EndoFLIP ${ }^{\circ}$ in gastroparesis. Rev Gastroenterol Mex. 2018;83(4):375-384. https://doi.org/10.1016/j.rgmx.2018.02.013

42. Desprez C, Melchior C, Wuestenberghs F, Zalar A, Jacques J, Leroi AM, et al. Pyloric distensibility measurement predicts symptomatic response to intrapyloric botulinum toxin injection. Gastrointest Endosc. 2019;90(5):754-760.e1. https://doi.org/10.1016/j.gie.2019.04.228

43. Tao J, Patel V, Mekaroonkamol P, Luo H, Li B, Guan $\mathrm{Q}$, et al. Technical Aspects of Peroral Endoscopic Pyloromyotomy. Gastrointest Endosc Clin N Am. 2019;29(1):117-126. https://doi.org/10.1016/j.giec.2018.08.012

44. Vosoughi K, Ichkhanian Y, Jacques J, Aadam AA, Benias PC, Law R, et al. Role of endoscopic functional luminal imaging probe in predicting the outcome of gastric peroral endoscopic pyloromyotomy (with video). Gastrointest Endosc. 2020 Jun;91(6): 1289-1299. https://doi.org/10.1016/j.gie.2020.01.044

45. Jehangir A, Malik Z, Petrov RV, Parkman HP. EndoFLIP and Pyloric Dilation for Gastroparesis Symptoms Refractory to Pyloromyotomy/Pyloroplasty. Dig Dis Sci. 2020. https://doi.org/10.1007/s10620-020-06510-0

46. Ilczyszyn A, Botha AJ. Feasibility of esophagogastric junction distensibility measurement during Nissen fundoplication. Dis Esophagus. 2014;27(7):637-44. https://doi.org/10.1111/dote.12130

47. Kim MP, Meisenbach LM, Chan EY. Tailored Fundoplication With Endoluminal Functional Lumen Imaging Probe Allows for Successful Minimally Invasive Hiatal Hernia Repair. Surg Laparosc Endosc Percutan Tech. 2018;28(3):178-182.

https://doi.org/10.1097/SLE.0000000000000527
48. DeHaan RK, Davila D, Frelich MJ, Gould JC. Esophagogastric junction distensibility is greater following Toupet compared to Nissen fundoplication. Surg Endosc. 2017;31(1):193-198. https://doi.org/10.1007/s00464-016-4956-0

49. Su B, Novak S, Callahan ZM, Kuchta K, Carbray J, Ujiki MB. Using impedance planimetry $\left(\right.$ EndoFLIP $\left.^{\mathrm{T \mu}}\right)$ in the operating room to assess gastroesophageal junction distensibility and predict patient outcomes following fundoplication. Surg Endosc. 2020;34(4):1761-1768. https://doi.org/10.1007/s00464-019-06925-5

50. Yu JX, Baker JR, Watts L, Varban OA, Chen JW, Rubenstein JH, et al. Functional Lumen Imaging Probe Is Useful for the Quantification of Gastric Sleeve Stenosis and Prediction of Response to Endoscopic Dilation: a Pilot Study. Obes Surg. 2020;30(2):786-789. https://doi.org/10.1007/s11695-019-04105-x

51. Liacouras CA, Furuta GT, Hirano I, Atkins D, Attwood SE, Bonis PA, et al. Eosinophilic esophagitis: updated consensus recommendations for children and adults. J Allergy Clin Immunol. 2011;128(1):3-20.e6; quiz 21-2. https://doi.org/10.1016/j.jaci.2011.02.040

52. Schoepfer AM, Safroneeva E, Bussmann C, Kuchen T, Portmann S, Simon HU, et al. Delay in diagnosis of eosinophilic esophagitis increases risk for stricture formation in a time-dependent manner. Gastroenterology. 2013;145(6):1230-6.e1-2. https://doi.org/10.1053/j.gastro.2013.08.015

53. Hirano I, Aceves SS. Clinical implications and pathogenesis of esophageal remodeling in eosinophilic esophagitis. Gastroenterol Clin North Am. 2014;43(2):297-316. https://doi.org/10.1016/j.gtc.2014.02.015

54. Chen JW, Pandolfino JE, Lin Z, Ciolino JD, Gonsalves N, Kahrilas PJ, et al. Severity of endoscopically identified esophageal rings correlates with reduced esophageal distensibility in eosinophilic esophagitis. Endoscopy. 2016;48(9):794-801. https://doi.org/10.1055/s-0042-107340

55. Carlson DA, Hirano I, Zalewski A, Gonsalves N, Lin Z, Pandolfino JE. Improvement in Esophageal Distensibility in Response to Medical and Diet Therapy in Eosinophilic Esophagitis. Clin Transl Gastroenterol. 2017;8(10):e119. https://doi.org/10.1038/ctg.2017.47

56. Kappelle WF, Bogte A, Siersema PD. Hydraulic dilation with a shape-measuring balloon in idiopathic achalasia: a feasibility study. Endoscopy. 2015;47(11):1028-34. https://doi.org/10.1055/s-0034-1392481

57. Baumann AJ, Carlson DA. EsoFLIP for esophageal dilation: proposed advantages. Curr Opin Gastroenterol. 2020;36(4):329-335. https://doi.org/10.1097/MOG.0000000000000639

58. O’Dea J, Siersema PD. Esophageal dilation with integrated balloon imaging: initial evaluation in a porcine model. Therap Adv Gastroenterol. 2013;6(2):109-14. https://doi.org/10.1177/1756283X12467566 
59. Nicodème F, Hirano I, Chen J, Robinson K, Lin Z, Xiao $\mathrm{Y}$, et al. Esophageal distensibility as a measure of disease severity in patients with eosinophilic esophagitis. Clin Gastroenterol Hepatol. 2013;11(9):1101-1107.e1. https://doi.org/10.1016/j.cgh.2013.03.020

60. Teitelbaum EN, Boris L, Arafat FO, Nicodème F, Lin Z, Kahrilas PJ, et al. Comparison of esophagogastric junction distensibility changes during POEM and Heller myotomy using intraoperative FLIP. Surg Endosc. 2013;27(12):4547-55. https://doi.org/10.1007/s00464-013-3121-2

61. Wu PI, Szczesniak MM, Craig PI, Choo L, Engelman J, Terkasher B, et al. Novel Intra-Procedural Distensibility Measurement Accurately Predicts Immediate Outcome of Pneumatic Dilatation for Idiopathic Achalasia. Am J
Gastroenterol. 2018;113(2):205-212.

https://doi.org/10.1038/ajg.2017.411

62. Sloan JA, Triggs JR, Pandolfino JE, Dbouk M, Brewer Gutierrez OI, El Zein M, et al. Treatment experience with a novel 30-mm hydrostatic balloon in esophageal dysmotility: a multicenter retrospective analysis. Gastrointest Endosc. 2020;92(6):1251-1257. https://doi.org/10.1016/j.gie.2020.04.076

63. Schnurre L, Murray FR, Schindler V, Runggaldier D, Fischbach L, Bordier V, et al. Short-term outcome after singular hydraulic EsoFLIP dilation in patients with achalasia: A feasibility study. Neurogastroenterol Motil. 2020;32(9):e13864.

https://doi.org/10.1111/nmo.13864 